\title{
BMJ Open Cohort profile: China respiratory illness surveillance among pregnant women (CRISP), 2015-2018
}

To cite: Chen L, Zhou S, Zhang Z, et al. Cohort profile: China respiratory illness surveillance among pregnant women (CRISP), 2015-2018. BMJ Open 2018;8:e19709. doi:10.1136/ bmjopen-2017-019709

- Prepublication history and additional material for this paper are available online. To view these files, please visit the journal online (http://dx.doi. org/10.1136/bmjopen-2017019709).

LC, SZ, ZZ and YW contributed equally.

Received 20 September 2017 Revised 7 February 2018 Accepted 16 February 2018

Check for updates

${ }^{1}$ Suzhou Center for Disease Control and Prevention, Suzhou, China

${ }^{2}$ Influenza Division, Centers for Disease Control and Prevention, Atlanta, Georgia, USA

${ }^{3}$ Suzhou Municipal Hospital, Suzhou, China

${ }^{4}$ Wuzhong Maternal and Child Health Care Institute, Suzhou, China

Correspondence to

Dr Jun Zhang;

sz_zhangj@163.com

\section{ABSTRACT}

Purpose We established the China Respiratory IIIness Surveillance among Pregnant women (CRISP) to conduct active surveillance for influenza-associated respiratory illness during pregnancy in China from 2015 to 2018. Among annual cohorts of pregnant women, we assess the incidence of acute respiratory illness (ARI), influenza-like illness (ILI), laboratory-confirmed influenza virus infection and the seroconversion proportion during the winter influenza season. We also plan to examine the effect of influenza virus infection on adverse pregnancy, delivery and infant health outcomes with cumulative data from the three annual cohorts.

Participants Cohort nurses enrol pregnant women in different trimesters of pregnancy from prenatal care facilities in Suzhou, Jiangsu Province, eastern China. Pregnant women who plan to deliver in the study facilities are eligible. Pregnant women who are seeking care for anything other than routine prenatal care, such as confirmation of low progesterone and threatened miscarriage, are excluded. At enrolment, study nurses collect baseline information on demographics, educationlevel attained, underlying medical conditions, seasonal influenza vaccination receipt, risk factors for influenza infection, gravidity and parity and contact information. For each participant, cohort nurses conduct twice weekly follow-up contacts, one phone call and one WeChat message (free instant messaging), from the time of enrolment until delivery or termination of pregnancy. During follow-up, study nurses ask about symptoms, timing and characteristics of ARI, healthcare-seeking behaviour and medications taken for participants reporting respiratory illness since the last contact. In addition, we collect combined nasal and throat swabs for identified ARI to test for influenza viruses. We collect paired sera before and after the influenza season. Active respiratory illness surveillance and seroinfection data during pregnancy of participants are linked to their medical record and the Suzhou Maternal Child Information System for detailed information on clinical treatment for respiratory illness, pregnancy, delivery and infant health outcomes.

Findings to date In 2015-2016, of 4915 pregnant women approached, 192 (4\%) refused to participate, 91 $(2 \%)$ were ineligible because they did not plan to deliver in one of the study hospitals or because their visit was for anything other than routine prenatal care and 4632 (94\%) were enrolled, $46 \%$ during their first trimester of

\section{Strengths and limitations of this study}

- Twice weekly active follow-up contacts increase the likelihood of capturing most illness episodes early to allow for sampling for respiratory viruses during a window of time when real time reverse transcription polymerase chain reaction is most likely to identify shed viruses.

- The Maternal Child Information System in Suzhou includes maternal and child healthcare information from all health facilities in Suzhou, allowing us to obtain documented birth outcome data for participants who deliver in any hospital within Suzhou.

- Most cohort nurses were retired, clinical nurses with experience establishing relationships and trust with patients, likely contributing to high cohort retention rates. The use of a popular social mobile application, WeChat, for active surveillance facilitated rapid, convenient communication between nurses and participants, likely increasing contact and sample collection rates.

- This study cannot assess influenza illness burden among pregnant women prior to five weeks gestation, usually the earliest gestation for pregnancy confirmation.

- The pregnant women enrolled in our study resided in an economically developed city, had a higher education level than those who declined to participate and may not represent pregnant women populations in rural areas of China.

pregnancy (range 5-12 weeks), 48\% during the second trimester (range 13-27 weeks) and 6\% during the third trimester (range 28-37 weeks). The median age of the enrollees was 27 years (range $16-45)$ and two $(0.04 \%$, $95 \% \mathrm{Cl} 0.01 \%$ to $0.17 \%$ ) reported influenza vaccination in the previous 12 months before pregnancy, while zero reported influenza vaccination in the previous 12 months during pregnancy. During the observation time of 648518 person-days, $1355 \mathrm{ARI}$ episodes were identified. Among 1127 swabs collected (for $83 \%$ of all ARIs), 68 (6\%) tested positive for influenza virus, for a laboratory-confirmed influenza incidence of 0.31 (95\% Cl 0.25 to 0.40$)$ per 100 person-months during pregnancy in the study cohort. Future plans Results will be used to describe influenza disease burden in this population to model potential 
numbers of influenza illnesses averted if influenza vaccination coverage were increased and to support enhanced influenza prevention and control strategies among pregnant women in China. We also plan to enrol and follow three cohorts of pregnant women over three influenza seasons during 2015-2018 which will allow an analysis of the effect of influenza virus infection during pregnancy on adverse pregnancy, delivery and infant outcomes.

\section{INTRODUCTION}

Globally, epidemiological studies have documented adverse outcomes with severe respiratory infections in pregnant women, including excess maternal mortality and miscarriage during pandemics such as the 2009 influenza $\mathrm{A}(\mathrm{H} 1 \mathrm{~N} 1)$ pandemic $\left(\mathrm{A}(\mathrm{H} 1 \mathrm{~N} 1)\right.$ pdm09). ${ }^{1-8}$ The few studies that have investigated influenza virus infections among pregnant women during seasonal epidemics have also observed that hospitalised pregnant women with respiratory illness have significantly longer lengths of stay and higher odds of delivery complications than hospitalised pregnant women without respiratory illness. ${ }^{9}$ Although several population-based studies suggested that only severe illness with $\mathrm{A}(\mathrm{H} 1 \mathrm{~N} 1) \operatorname{pdm} 09$, and not mild illness with $\mathrm{A}(\mathrm{H} 1 \mathrm{~N} 1)$ or any illness with seasonal influenza, was associated with increased risk of preterm birth and fetal death, these studies often lacked laboratory-confirmation of influenza, were limited to high-income countries and relied on retrospective testing and data collection by health facilities, leading to the potential for misclassified diagnoses. ${ }^{10}$ Thus, evidence demonstrating the risk of seasonal influenza-associated infection, illness and the severity of illness among pregnant women and impact of influenza infection on maternal and child health outcomes at the population level is insufficient. Further research is needed to establish the burden and effect of influenza virus infection during pregnancy, especially in low-income and middle-income countries.

The WHO recommends seasonal influenza immunisation for pregnant women during any trimester to prevent influenza virus infection. ${ }^{11}$ Although the Chinese Center for Disease Prevention and Control (China CDC) endorsed this recommendation in the 2014-2015 technical guidelines for the prevention and control of seasonal influenza, ${ }^{12}$ the influenza vaccine is not included in the national immunisation programme. Conflicting recommendations within different government sectors have further hindered introduction of influenza vaccination among pregnant women in China. In the latest version of the Chinese Pharmacopeia published in 2015, pregnancy was listed as one of the contraindications to receiving seasonal influenza vaccination. ${ }^{13}$ A knowledge, attitudes and practices survey on influenza vaccination among pregnant women conducted in Suzhou City, China, in 2013, found that none of the 1600 participants surveyed received the seasonal influenza vaccine during their pregnancy. ${ }^{14}$ In the same survey, $61 \%$ of the pregnant women interviewed wanted to know how to prevent and control influenza. ${ }^{15}$
In China, local evidence related to influenza disease burden among pregnant women is limited, and awareness about influenza vaccine effectiveness is deficient among both pregnant women and healthcare providers. ${ }^{16}$ Disease and economic burden data for seasonal influenza in China along with local data on seasonal influenza vaccine effectiveness and safety among pregnant women are needed to inform and promote policy. ${ }^{17}$ This study collects influenza disease burden data among pregnant women in China, which may inform the development of influenza prevention and control strategies in this population.

In October 2015, we established China Respiratory Illness Surveillance among Pregnant women (CRISP), annual cohorts of pregnant women enrolled at different gestational ages, to conduct active surveillance for influenza-associated respiratory illness among pregnant women in China. Our primary objectives were to: (1) estimate the incidence of acute respiratory illness (ARI), influenza-like illness (ILI) and laboratory-confirmed influenza virus infection in the study cohort; (2) estimate the incidence of hospitalisations associated with ARI in the study cohort and (3) estimate the influenza seroconversion proportion among pregnant women during the winter influenza season. The secondary objectives were to: (1) examine the effect of influenza virus infection during pregnancy on adverse pregnancy, delivery and infant outcomes and (2) explore risk factors associated with severe seasonal influenza virus illness among pregnant women. We will meet our secondary objectives by combining multiple years of data from CRISP.

\section{COHORT DESCRIPTION}

\section{Study setting and population}

This study is being implemented in Suzhou, a major city in the Yangtze Delta with a 2015 population of 10.62 million, located in the southeast region of Jiangsu Province in eastern China (figure 1). The gross domestic product per capita in Suzhou was US\$21 000 (calculated by resident population) in 2015. ${ }^{18}$ Suzhou consists of five municipal districts (Gusu District, New and High-tech District, Wuzhong District, Xiangcheng District and Industrial Park District) and five county-level districts (figure 1), with a total land area of $8488 \mathrm{~km}^{2}$.

Suzhou's well-established system for maternal and child healthcare includes prepregnancy services with pregnancy planning counselling, reproductive health education and early pregnancy confirmation in prenatal healthcare facilities. Premarital health check-up clinics within district marriage registration centres also provide early pregnancy confirmation services. More than $90 \%$ of resident couples obtain premarital health check-ups prior to getting their marriage registration, and among women examined, more than $10 \%$ has a positive pregnancy test. Pregnancy refers to the state of carrying a developing embryo or fetus within the female body, which is usually identified by positive results on an over-the-counter urine 


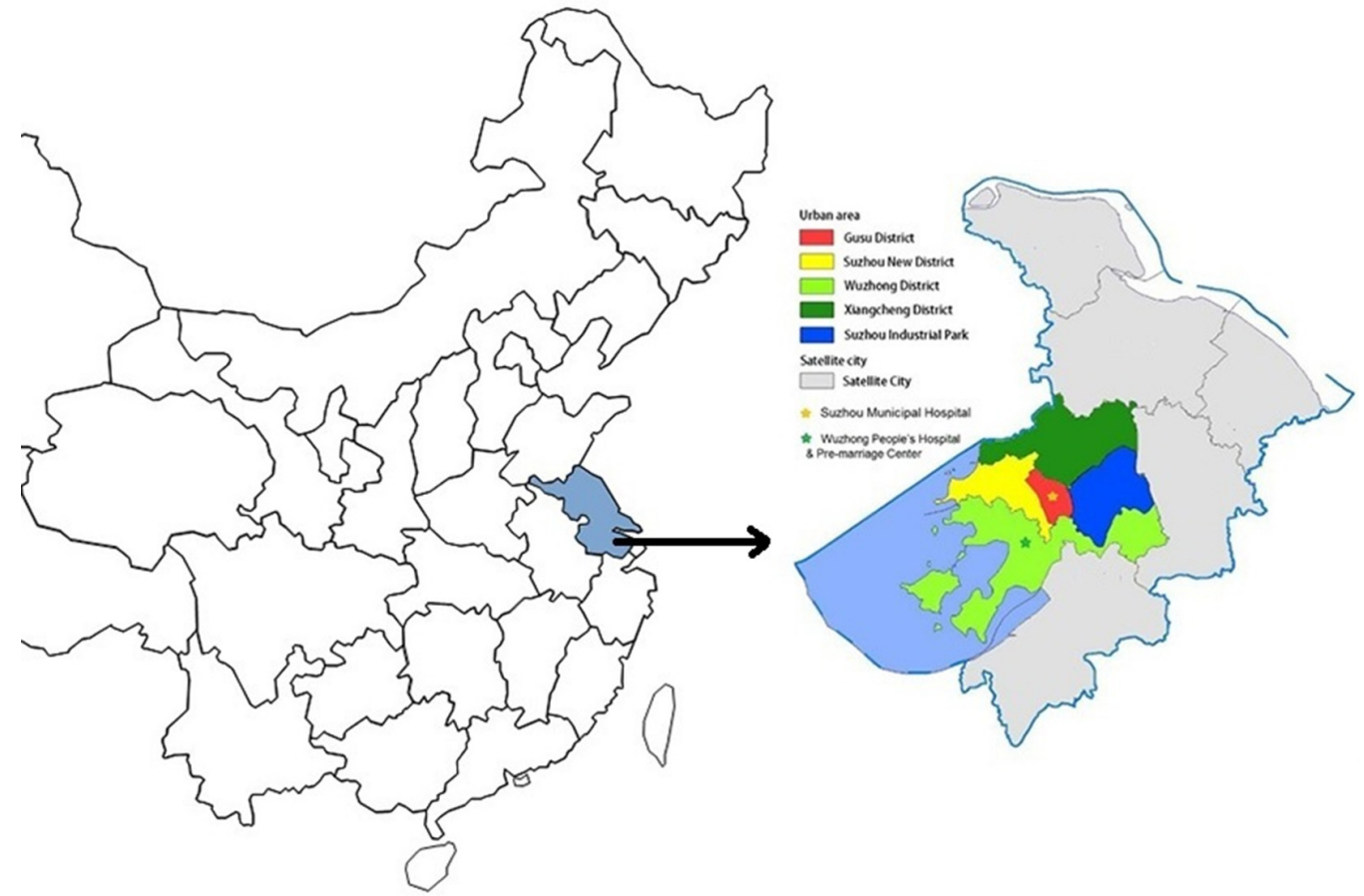

Figure 1 Map of city jurisdiction and study facilities for China Respiratory Illness Surveillance among Pregnant women (CRISP) in Suzhou, Jiangsu Province, China.

test, and confirmed through a blood test, ultrasound and detection of fetal heartbeat. During pregnancy, 13 recommended routine prenatal check-ups, three regular post-partum check-ups and seven regular newborn/ toddler visits for all children up to 3 years of age are provided in prenatal healthcare facilities in Suzhou, with more check-ups recommended for high-risk populations. Approximately $46 \%$ of pregnant women have nine or more prenatal care visits, ${ }^{19}$ and more than $98 \%$ children have more than one newborn/toddler health check-up. ${ }^{20}$ Suzhou has a Maternal Child Information System (MCIS) which is a comprehensive electronic information platform that stores all of the above-mentioned prenatal check-up records, delivery records and infant/child healthcare records. Information stored includes pregnancy, delivery and infant growth and developmental outcomes such as complications (gestational diabetes and hypertensive disorders of pregnancy), live versus stillbirth, gestational age at birth, delivery method (vaginal vs caesarean), birth weight, Apgar score, any recorded birth defect(s), infant peripheral blood haemoglobin concentration at 6 months, infant height and weight and infant developmental screening.

In 2012 , more than $95 \%$ of pregnant women who had a live or stillbirth delivery after 28 weeks gestation in Suzhou had received at least one recommended prenatal care visit, more than $99 \%$ delivered at a healthcare facility and $100 \%$ of pregnant women who received prenatal care in Suzhou were included in the MCIS system. Similar to other cities throughout China, Suzhou City has three tiers of maternal healthcare facilities, including 15 tertiary facilities, 31 secondary facilities and 54 primary level facilities $^{21}$ typically located within hospitals or clinics.
Tertiary facilities have the highest capacity for medical services, management and research. In 2014, $7 \%$ of pregnant women chose prenatal care and delivery services at Suzhou primary level facilities, $38 \%$ at secondary facilities and $55 \%$ at tertiary facilities. ${ }^{21}$

In this study, we recruited pregnant women from three maternal healthcare facilities: one tertiary facility in Suzhou Municipal Hospital, one secondary facility in Wuzhong People's Hospital and the premarriage health check-up centre in Wuzhong District (figure 2). Both Suzhou Municipal Hospital and Wuzhong People's Hospital had existing influenza surveillance systems.

Suzhou Municipal Hospital, located in the central area of Gusu District, had 17415 deliveries in 2014. Among pregnant women who delivered at Suzhou Municipal Hospital, 93\% were municipal district residents. Wuzhong People's Hospital, located in Wuzhong District, had 3118 deliveries in 2014. Among pregnant women who delivered at this hospital, $100 \%$ were from municipal districts and 99\% were from Wuzhong District. Among all deliveries in Suzhou in 2014, 17\% were at either Suzhou Municipal Hospital or Wuzhong People's Hospital (table 1). We selected Wuzhong District premarriage health check-up centre to increase the enrolment of pregnant women in their first trimester who planned to deliver at Wuzhong People's Hospital or Suzhou Municipal Hospital.

Pregnant women were eligible for inclusion if they: (1) were seeking pregnancy confirmation or prenatal care services in one of the study facilities and (2) planned to deliver in a study facility. Pregnant women who were seeking care for anything other than routine prenatal care, such as confirmation of low progesterone and threatened miscarriage, were excluded. 


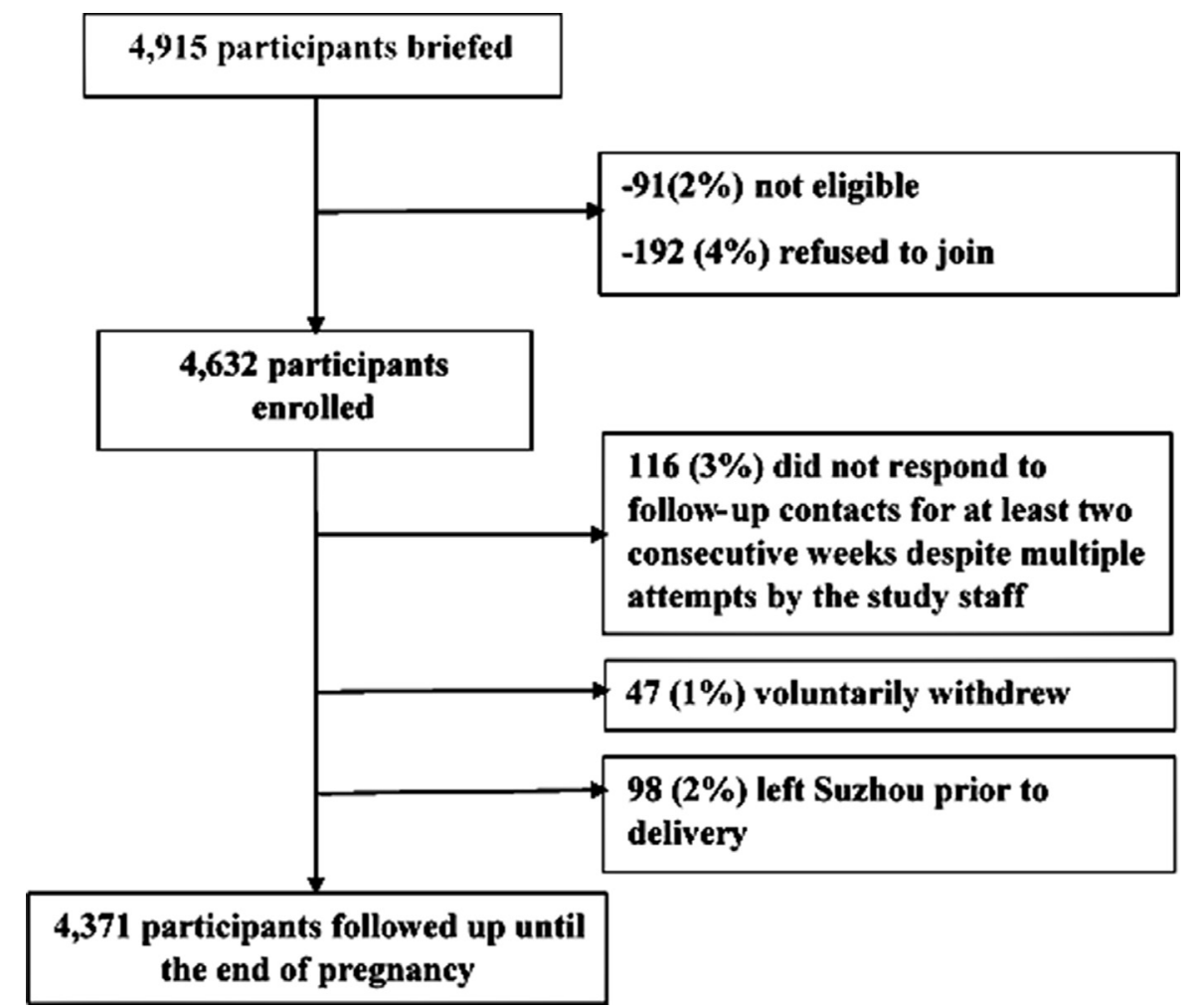

Figure 2 Flow chart of participant profile.

WHO has estimated that approximately $5 \%-10 \%$ of adults will develop influenza virus infection annually. ${ }^{11}$ To estimate sample size, we used a conservative $3 \%$ influenza illness attack rate for pregnant women during the surveillance period, with $\alpha=0.05(\mathrm{Za}=1.96)$ and error $\mathrm{d}=0.2 \mathrm{p}$. Thus, to detect an influenza illness attack rate of $3 \%$ in

Table 1 Live births in Suzhou and in study facilities by registered current address of pregnant woman in 2014

\begin{tabular}{|c|c|c|c|}
\hline $\begin{array}{l}\text { Residential district } \\
\text { or county }\end{array}$ & $\begin{array}{l}\text { No. of live } \\
\text { births in } \\
2014 \text { in } \\
\text { Suzhou }\end{array}$ & $\begin{array}{l}\text { No. of live } \\
\text { births in } \\
\text { Suzhou } \\
\text { Municipal } \\
\text { Hospital }\end{array}$ & $\begin{array}{l}\text { No. of live } \\
\text { births in } \\
\text { Wuzhong } \\
\text { People's } \\
\text { Hospital }\end{array}$ \\
\hline \multicolumn{4}{|l|}{ Municipal district } \\
\hline Gusu & 8470 & 3496 & 1 \\
\hline $\begin{array}{l}\text { New and High- } \\
\text { tech }\end{array}$ & 7397 & 2175 & 0 \\
\hline Wuzhong & 13438 & 4000 & 3117 \\
\hline Xiangcheng & 10972 & 2225 & 0 \\
\hline Industrial Park & 11791 & 4299 & 0 \\
\hline \multicolumn{4}{|l|}{ County } \\
\hline Changshu & 14483 & 217 & 0 \\
\hline Kunshan & 19306 & 304 & 0 \\
\hline Wujiang & 14715 & 575 & 0 \\
\hline Taicang & 7268 & 73 & 0 \\
\hline Zhangjiagang & 13752 & 51 & 0 \\
\hline Total & 121592 & 17415 & 3118 \\
\hline
\end{tabular}

our cohort, we required a sample size of $\mathrm{N}=\mathrm{Za}^{2} * \mathrm{p} *(1-\mathrm{p}) /$ $\mathrm{d}^{2}=3233$. We estimated that $10 \%$ of participants would be lost to follow-up after enrolment, leading to a sample size of approximately 3600 pregnant women to follow during the winter influenza peak. As we aimed to measure influenza infection incidence during the summer influenza peak also, and to maintain a similar pregnant women cohort size with similar numbers of women in each trimester of pregnancy, we continued to enrol approximately 100 first trimester pregnant women every month after the intensive initial enrolment in October, before the winter influenza peak.

\section{Design and data collection}

Enrolment (complete for year 1 and year 2, ongoing for year 3)

We enrolled pregnant women at different trimesters from the prenatal care departments in study facilities during October 2015, enrolling most of the cohort prior to the local winter influenza season which usually begins in November. However, to ensure enrolment of adequate numbers of pregnant women in their first trimester and to ensure sufficient numbers of pregnant women to follow during the summer influenza season, we continuously enrolled first trimester pregnant women from the pregnancy confirmation clinics in the two hospitals and the Wuzhong premarriage health check centre from October 2015 through September 2016, allowing us to maintain observation throughout the year. Using the same approach, we enrolled the majority of the second cohort of pregnant women in October 2016 and continued to enrol first trimester pregnant women through September 
2017. We enrolled the majority of a third cohort of pregnant women in October 2017, and we will continue to enrol first trimester pregnant women for this third cohort through September 2018.

We informed participants about the study prior to asking for written consent. We conducted face-to-face interviews using a structured questionnaire (Annex 1). Every participant received an enrolment card with the name and contact information of their designated study nurse, along with a digital thermometer. Study nurses educated and encouraged participants to use the enrolment card to facilitate prenatal care services and respiratory illness consultation in study hospitals. The enrolment card allowed participants with respiratory illness to receive expedited healthcare services in the study hospitals' respiratory clinics, shortening waiting times from up to 3 hours to less than $30 \mathrm{~min}$ and may have incentivised women with respiratory symptoms to seek care at a study hospital.

\section{Active surveillance for illness (complete for year 1 and year 2 ongoing for year 3)}

Active surveillance for acute illness consisted of 2 weekly contacts from study nurses starting at the date of enrolment and continuing through delivery or end of pregnancy/loss of pregnancy: (1) a weekly telephone call by study nurses using a standard questionnaire (Annex 2) which asked about illnesses since the last contact and (2) one WeChat text message (via their cellular telephone). WeChat is a free, instant messaging application that is widely used in China, particularly among younger populations. The weekly WeChat text message asked the recipient: 'Have you had any illness since I last contacted you-yes or no?' If a participant replied 'yes', the study nurse would call the participant.

The follow-up aimed to capture ARI episodes and, for all respiratory illness episodes, to collect combined nasal and throat swabs within 72 hours of illness onset. When a current or recent illness was identified by either method, the nurse asked for date of illness onset, symptoms (including cough, sore throat, stuffy nose, chest pain, difficulty breathing, feeling feverish, having a measured temperature $\geq 38^{\circ} \mathrm{C}$ (and if so, the highest temperature measured), chills or headache), severity of illness, illness duration, functional impairment, use of over the counter drugs and whether or not the participant visited any outpatient clinic or was admitted to any hospital.

When pregnant women experienced an ARI, defined as onset of at least one respiratory symptom (cough, sore throat, stuffy nose, chest pain or difficulty breathing) and at least one systemic symptom (feeling feverish or having a measured temperature $\geq 38^{\circ} \mathrm{C}$, chills or headache) or at least two respiratory symptoms, we encouraged them to visit the study hospital's respiratory illness department (ambulatory clinics) within 24 hours of contact. The broad definition for ARI allowed us to compare the sensitivity of various illness definitions for influenza infection. Study nurses informed the physician on duty to expect a study participant's visit and reminded the study participant to show her enrolment card on arrival at the clinic to receive expedited services. We trained all physicians in the respiratory illness clinic to collect combined nasal and throat swabs $^{22}$ for enrolled pregnant women during their visits. If an enrolled pregnant woman did not wish to visit one of the two study hospitals, the study nurse conducted a household visit to collect the combined nasal and throat swab. The physicians and study nurses followed standard procedures to collect the respiratory specimens following the national influenza surveillance guidelines. ${ }^{23}$

\section{Loss to follow-up}

An enrolled participant was considered to have complete data if she completed the enrolment interview, responded to weekly surveillance contacts by study nurses until delivery or termination of pregnancy, and had perinatal records available for review.

\section{Sera collection starting in year 2}

The Suzhou Maternal and Child Health system offers six routine prenatal check-ups that include a blood draw. The first blood draw in week 12 tests for complete blood count, blood type, blood chemistries and HIV/rubella/ hepatitis B surface antigen. The following blood draws screen for Down Syndrome (week 16), test for complete blood count (weeks 22 and 37) and blood chemistries (week 30) and screen for diabetes (week 26). ${ }^{21}$ Most blood draws have excess sera remaining after prenatal care facilities conduct routine testing. Laboratories at the study hospitals and the premarital clinic store excess sera samples from all blood draws at $4^{\circ} \mathrm{C}$ for 1 week. Starting in the second year of our study, dedicated nurses identified excess sera drawn from enrolled pregnant women within 2 days of their routine prenatal blood draws from study facility laboratories in October, the month prior to the start of the winter influenza season. We used these sera as the preinfluenza season sera sample for a future paired sera analysis. From November to May, study nurses screened excess sera for samples from enrolled participants to obtain the delivery or postseason sera specimen. Using this approach, we collected paired sera samples from leftover sera of blood drawn for routine maternal care; we collected the first sera sample near the time of enrolment and prior to the winter influenza season and the second sample at the time of delivery, pregnancy termination or, if the woman was still pregnant, in May, after the winter influenza season.

\section{Data linkage}

Participants' active respiratory illness surveillance data and seroinfection results during pregnancy will be linked to their medical records and the MCIS using identity numbers from Chinese government-issued identification cards or, when identity numbers are missing, using names and dates of birth. The MCIS, managed by the Suzhou Health Bureau, provides a unified electronic data input platform for all maternal and child healthcare facilities in Suzhou, including community healthcare centres, allowing us to 
Table 2 Data and samples collected during three data collection periods for each annual cohort in China Respiratory Illness Surveillance among Pregnant women (CRISP), Suzhou 2015-2018

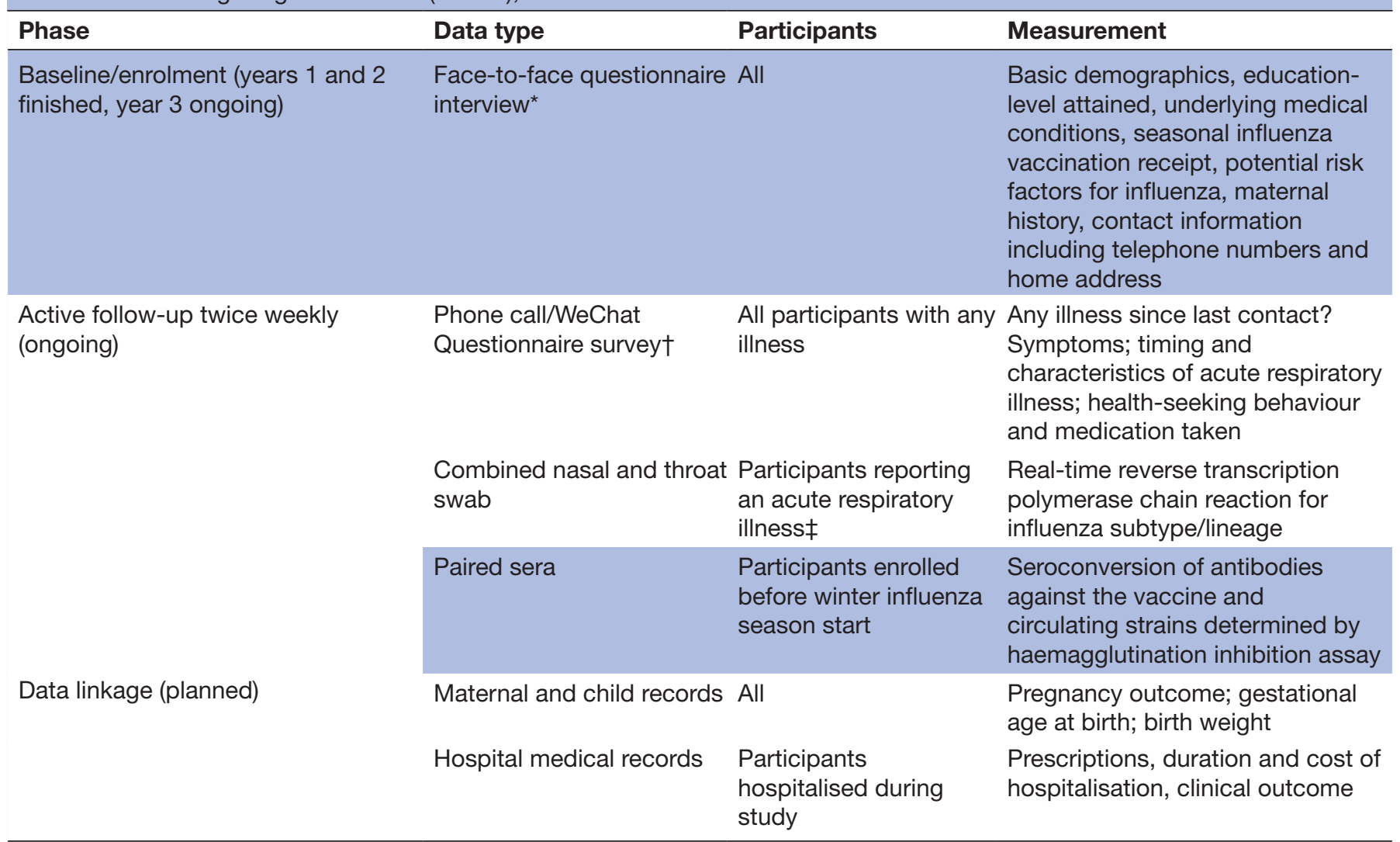

*See Annex 1.

†See Annex 2.

$\ddagger$ An acute respiratory illness refers to onset of at least one respiratory symptom (cough, sore throat, stuffy nose, chest pain or difficulty breathing) and at least one systemic symptom (feeling feverish or having a measured fever $\geq 38^{\circ} \mathrm{C}$, chills or headache) or at least two respiratory symptoms.

obtain documented birth outcome data for all participants who deliver in any hospital within Suzhou. The Suzhou Maternal and Child Health Institute is responsible for training and monitoring all healthcare staff in Suzhou on the use of this platform, which has been shown to include data on all women delivering in Suzhou and their infants, though detailed information on the clinical course of delivery is lacking. ${ }^{24}$ More detailed birth outcome data can be captured directly from the Hospital Information System (HIS) in facilities providing delivery services. At the end of year 3, study nurses plan to review participants' maternal health medical records to gather information about treatment and outcomes of any respiratory diseases for pregnant women hospitalised during the study period. In addition, study nurses will collect data on women in all three cohorts related to adverse pregnancy, delivery and infant outcomes, including stillbirths, premature births and small for gestational age from the MCIS and the HIS in facilities where enrolled women delivered.

\section{What has been measured}

The data and biological samples collected during enrolment, active follow-up and post-follow-up phase are summarised in table 2.
The main clinical outcomes of interest in the first year were laboratory-confirmed influenza associated ARI, ILI and severe acute respiratory infection (SARI). We plan to compare incidence of influenza illness using alternative syndromic case definitions, such as the WHO case definitions for ILI and SARI in 2014, in our analysis. We defined ARI as described above. We defined ILI as measured temperature of $\geq 38^{\circ} \mathrm{C}$ and cough, with onset within the last 10 days. ${ }^{25}$ We defined SARI as history of fever or measured temperature of $\geq 38^{\circ} \mathrm{C}$ and cough, with onset within the last 10 days, requiring hospitalisation. ${ }^{25}$ All combined nasal and throat swab samples were analysed using real-time reverse transcription polymerase chain reaction (rRT-PCR) to test for influenza virus by subtype/lineage in the Suzhou CDC laboratory. We plan to perform serological tests of paired sera specimens using the haemagglutination inhibition assay to determine titres of antibodies against the vaccine and circulating strains of influenza A virus subtypes $\mathrm{H} 1$ and $\mathrm{H} 3$ and both influenza B lineages. A fourfold or greater rise in virus-specific antibody between paired sera, and a minimum second titre of 1:40, will establish seroconversion and will demonstrate influenza infection history during the study period. 
Table 3 Comparison between enrolled pregnant women and those who refused to participate in Suzhou, 2015-2016

\begin{tabular}{lrrr} 
& $\begin{array}{l}\text { Pregnant } \\
\text { women } \\
\text { enrolled } \\
\text { (Row \%) }\end{array}$ & $\begin{array}{l}\text { Pregnant } \\
\text { women } \\
\text { refused to } \\
\text { join (Row \%) }\end{array}$ & P values \\
\hline Age group & & & \\
15-24 & $791(95)$ & $42(5)$ & 0.25 \\
$25-29$ & $2671(96)$ & $116(4)$ & \\
$30-34$ & $921(97)$ & $29(3)$ & \\
35-39 & $249(98)$ & $5(2)$ & \\
Total & $4632(96)$ & $192(4)$ & \\
Education level & & & \\
$\begin{array}{l}\text { Junior high school or } \\
\text { lower (Grade 9) }\end{array}$ & $375(88)$ & $51(12)$ & \\
$\begin{array}{l}\text { Senior high school } \\
\text { (Grade 12) }\end{array}$ & $884(95)$ & $50(5)$ & \\
College or higher & $3357(97)$ & $91(3)$ & \\
\hline
\end{tabular}

The pregnancy, delivery and infant growth and developmental outcomes of interest include complications (gestational diabetes and hypertensive disorders of pregnancy), spontaneous abortions, live versus stillbirth, gestational age at birth, delivery method (vaginal vs caesarean), small for gestational age, Apgar score, any recorded birth defect(s), peripheral blood haemoglobin concentration at 6 months, height at 6 months, weight at 6 months and developmental screening at 6 months.

\section{FINDINGS TO DATE}

A flow chart of the participants profile is shown in figure 2. From 1 October 2015 to 30 September 2016, we approached 4915 pregnant women to participate in our study. Among these, 91 (2\%) did not meet inclusion criteria, 192 (4\%) refused to participate and 4632 consented to participate, resulting in a response rate of 96\% (4632/4824). Study nurses collected basic information on women who refused to participate, including age, highest education level attained and reasons for refusing to participate. Compared with pregnant women who refused to participate, enrolled pregnant women were of similar age $(\mathrm{p}=0.25)$, but were more likely to have at least a high school education $(\mathrm{p}<0.01)$. Twelve per cent of women with a junior high school education (Grade 9) or less refused participation compared with $5 \%$ of senior high school (Grade 12) graduates and $3 \%$ of college graduates, $\mathrm{p}<0.01$ (table 3 ). Compared with all pregnant women seeking prenatal healthcare in 2015 in Suzhou, the proportion of women aged 25-29 years enrolled in this study was higher, as was the proportion who had attained at least a college education level (table 4).

Among the 4632 cohort of pregnant women in the first year, $261(6 \%)$ did not have complete data at the end of the follow-up period: $116(3 \%)$ did not respond
Table 4 Baseline characteristics of respondents compared with local average of pregnant women in Suzhou, 2015

\begin{tabular}{|c|c|c|c|}
\hline & $\begin{array}{l}\text { Suzhou average } \\
\text { (Col. \%) }\end{array}$ & $\begin{array}{l}\text { Study cohort } \\
\text { (Col. \%) }\end{array}$ & $P$ values \\
\hline \multicolumn{4}{|l|}{ Age group } \\
\hline$<20$ & $2890(2.2)$ & $26(0.6)$ & \multirow[t]{6}{*}{$<0.001$} \\
\hline $20-24$ & $27388(20.8)$ & 765 (16.5) & \\
\hline $25-29$ & $63109(47.8)$ & $2671(57.7)$ & \\
\hline $30-34$ & $30030(22.8)$ & 921 (19.9) & \\
\hline$>35$ & $8595(6.5)$ & $249(5.4)$ & \\
\hline Total & 132012 & 4632 & \\
\hline \multicolumn{4}{|l|}{ Influenza vaccine } \\
\hline Vaccinated & $1 \% \dagger$ & $0.043 \%$ & \\
\hline \multicolumn{4}{|l|}{ Education level $(n=4616)$} \\
\hline $\begin{array}{l}\text { Primary school or } \\
\text { lower (Grade 6) }\end{array}$ & 3014 (2.3) & $18(0.4)$ & \multirow[t]{5}{*}{$<0.001$} \\
\hline $\begin{array}{l}\text { Junior high school } \\
\text { (Grade 9) }\end{array}$ & $29039(21.8)$ & $357(7.7)$ & \\
\hline $\begin{array}{l}\text { Senior high school } \\
\text { (Grade 12) }\end{array}$ & $28989(21.7)$ & $884(19.2)$ & \\
\hline College or higher & $72476(54.3)$ & $3357(72.7)$ & \\
\hline Total & 133518 & 4632 & \\
\hline
\end{tabular}

*Data source: Suzhou Maternal Child Information System (unpublished data).

†Data source: KAP study among 1600 pregnant women during 2012-2013 in Suzhou, China. ${ }^{15}$

to follow-up contacts for at least two consecutive weeks despite multiple attempts by the study staff; $47(1 \%)$ voluntarily withdrew from the study and 98 (2\%) left Suzhou prior to delivery. Table 5 displays the characteristics of pregnant women with and without complete information. While complete information was generally high across demographic groups, incomplete information was higher among women aged $<25$ years $(61 / 791,8 \%)$ and among those with junior high school (ninth grade) or less education $(32 / 375,9 \%)$.

Among the enrolled 4632 pregnant women, 46\% (2126) were enrolled during their first trimester of pregnancy (median 7 weeks, range 5-12weeks), 48\% (2214) during the second trimester (median 17 weeks, range 13-27 weeks) and $6 \%$ (292) during the third trimester (median 30 weeks, range 28-37weeks). The median age of enrollees was 27 years (range 16-45) and two $(0.04 \%$, 95\% CI $0.01 \%$ to $0.17 \%$ ) reported influenza vaccination in the previous 12 months before pregnancy, while zero reported influenza vaccination in the previous 12 months during pregnancy. During the observation time of 648518 person-days, 1355 ARI episodes were identified, including 79 ILI episodes of which 11 met the SARI case definition. Among 1127 swabs collected (for $83 \%$ of all ARIs), 68 (6\%) tested positive for influenza virus, for a laboratory-confirmed influenza incidence of 0.31 (95\% CI 0.25 to 0.40 ) per 100 person-months during pregnancy in the study cohort. 
Table 5 Characteristics of participants with and without complete information, Suzhou, 2015-2016

\begin{tabular}{lccc}
\hline & $\begin{array}{l}\text { Participants } \\
\text { with } \\
\text { complete } \\
\text { information } \\
\text { (Row \%) }\end{array}$ & $\begin{array}{l}\text { Participants } \\
\text { without } \\
\text { complete } \\
\text { information } \\
\text { (Row \%) }\end{array}$ & P values \\
\hline Age group & $730(92)$ & $61(8)$ & 0.005 \\
\hline 15-24 & $2521(94)$ & $150(6)$ & \\
$25-29$ & $884(96)$ & $37(4)$ & \\
30-34 & $218(94)$ & $13(6)$ & \\
35-39 & $18(100)$ & $0(0)$ & \\
40-49 & 4371 & 261 & \\
Total & 1 & 1 & \\
Influenza vaccine & & $32(9)$ & \\
\hline $\begin{array}{l}\text { Vaccinated } \\
\text { Education level }\end{array}$ & $343(91)$ & $61(7)$ & \\
\hline $\begin{array}{l}\text { Junior high school or } \\
\text { lower (Grade 9) }\end{array}$ & & & \\
$\begin{array}{l}\text { Senior high school } \\
\text { (Grade 12) }\end{array}$ & $823(93)$ & $167(5)$ & \\
College or higher & $3190(95)$ & & \\
\hline
\end{tabular}

We presented mid-term results through the end of February 2016 at Options IX for the Control of Influenza Conference. $^{26}$

\section{Strengths and limitations}

Twice weekly follow-up contacts increase the likelihood of capturing the majority of illness episodes. In addition, twice weekly follow-up captures illness early and allows for sampling for respiratory viruses during a window of time when PCR is most likely to identify shed viruses. This prospective surveillance cohort uses laboratory-confirmed influenza as the outcome and therefore provides valuable data related to disease burden specific to influenza. The large cohort size (>4000women enrolled each year) will allow us to combine several years of data to examine the relationship between influenza illness and pregnancy outcomes. The Maternal Child Information System in Suzhou includes maternal and child healthcare information from all health facilities in Suzhou, allowing us to obtain documented birth outcome data for participants who deliver in any hospital within Suzhou.

Two additional strengths included the study personnel and the surveillance contact methods. Most cohort nurses were retired, clinical nurses with experience establishing relationships and trust with patients; their experience may have contributed to high cohort retention rates. Further, the use of a popular social mobile application, WeChat, for active surveillance likely contributed to the high contact rate and sample collection by facilitating rapid, convenient communication between nurses and participants.
This cohort has several limitations. First, we may not identify all influenza infections through active surveillance since our case definitions do not capture atypical and asymptomatic manifestations of influenza virus infection. However, the serology component in the second and third years may address this limitation. In addition, our study cannot assess influenza illness burden among pregnant women prior to 5 weeks gestation, usually the earliest gestation that pregnant women are able to confirm pregnancy. Third, the power calculation we conducted a priori may only pertain to the influenza seroconversion estimates and not to the symptomatic influenza illness risk. Finally, Suzhou is an economically developed city in China, and the pregnant women enrolled in our study had a higher education level than those who declined to participate; therefore, they may not represent pregnant women populations in the rural areas of Jiangsu Province or other provinces in China. Among enrolled pregnant women, those aged $<25$ years and those with junior high school (Grade 9) education or less were more likely to have incomplete health-related information.

Acknowledgements We thank Chen Hong and Zhang Rui of the China-US Emerging and Re-emerging Infectious Diseases Program Office, Chinese Center for Disease Control and Prevention, for their program management support for this study.

Collaborators We welcome interested potential collaborators to contact us. Please send inquiries to JZ (sz_zhangj@163.com) and CMG (cqg4@cdc.gov).

Contributors LC, SZ, YS, CMG and JZ conceived and designed the study. LC, $\mathrm{ZZ}, \mathrm{YW}, \mathrm{LB}, \mathrm{YT}$ and FS implemented the data collection; LB and YT analysed the data; LC, SZ, YS, RZ, ADI, MGT and CMG wrote the draft of the manuscript. All authors helped revise the manuscript. All authors have seen and approved the final manuscript and have contributed significantly to the work.

Funding This work was supported by the Cooperative Agreement Number, 5U2GGH000018, funded by the US Centers for Disease Control and Prevention.

Disclaimer The findings and conclusions in this report are those of the authors and do not necessarily represent the official position of the Centers for Disease Control and Prevention.

Competing interests None declared.

Patient consent Not required.

Ethics approval This study was approved by the Institutional Review Board (IRB) of Jiangsu Provincial Center for Disease Control and Prevention; the US Centers for Disease Control and Prevention relied upon Jiangsu Provincial IRB approval.

Provenance and peer review Not commissioned; externally peer reviewed.

Data sharing statement Further information about the data can be obtained from the corresponding author, JZ (sz_zhangj@163.com).

Open Access This is an Open Access article distributed in accordance with the Creative Commons Attribution Non Commercial (CC BY-NC 4.0) license, which permits others to distribute, remix, adapt, build upon this work non-commercially, and license their derivative works on different terms, provided the original work is properly cited and the use is non-commercial. See: http://creativecommons.org/ licenses/by-nc/4.0/

(c) Article author(s) (or their employer(s) unless otherwise stated in the text of the article) 2018. All rights reserved. No commercial use is permitted unless otherwise expressly granted.

\section{REFERENCES}

1. ANZIC Influenza Investigators and Australasian Maternity Outcomes Surveillance System. Critical illness due to 2009 A/H1N1 influenza in pregnant and postpartum women: population based cohort study. BMJ 2010;340:751. 
2. Centers for Disease Control and Prevention (CDC). Maternal and infant outcomes among severely ill pregnant and postpartum women with 2009 pandemic influenza A (H1N1) - United States, April 2009August 2010. MMWR Morb Mortal Wkly Rep 2011;60:1193-6.

3. Creanga AA, Johnson TF, Graitcer SB, et al. Severity of 2009 pandemic influenza $A(\mathrm{H} 1 \mathrm{~N} 1)$ virus infection in pregnant women. Obstet Gynecol 2010;115:717-26.

4. Hewagama S, Walker SP, Stuart RL, et al. 2009 H1N1 influenza A and pregnancy outcomes in Victoria, Australia. Clin Infect Dis 2010;50:686-90.

5. Knight M, Pierce M, Seppelt I, et al. Critical illness with AH1N1v influenza in pregnancy: a comparison of two population-based cohorts. BJOG 2011;118:232-9.

6. Louie JK, Acosta M, Jamieson DJ, et al. Severe 2009 H1N1 influenza in pregnant and postpartum women in California. N Engl J Med 2010;362:27-35.

7. Oluyomi-Obi T, Avery L, Schneider C, et al. Perinatal and maternal outcomes in critically ill obstetrics patients with pandemic H1N1 Influenza A. J Obstet Gynaecol Can 2010;32:443-47.

8. Siston AM, Rasmussen SA, Honein MA, et al. Pandemic 2009 influenza $A(\mathrm{H} 1 \mathrm{~N} 1)$ virus illness among pregnant women in the United States. JAMA 2010;303:1517.

9. Cox S, Posner SF, McPheeters M, et al. Hospitalizations with respiratory illness among pregnant women during influenza season. Obstet Gynecol 2006;107:1315-22.

10. Fell DB, Savitz DA, Kramer MS, et al. Maternal influenza and birth outcomes: systematic review of comparative studies. BJOG 2017;124:48-59.

11. World Health Orgnization. Vaccines against influenza WHO position paper - November 2012. Wkly Epidemiol Rec 2012;87:461-76.

12. Feng $L$, Yang $P$, Zhang $T$, et al. Technical guidelines for the application of seasonal influenza vaccine in China (2014-2015). Hum Vaccin Immunother 2015;11:2077-101.

13. Chinese Pharmacopoeia Commission. The pharmacopoeia of the People's Republic of China. Beijing: China Medical Science Press, 2015.

14. Song $Y$, Zhang $T$, Chen $L$, et al. Increasing seasonal influenza vaccination among high risk groups in China: Do community healthcare workers have a role to play? Vaccine 2017;35:4060-3.
15. Chen L, Zhang J, Zu R, et al. Influenza-related knowledge, attitudes, and practices among pregnant women: a cross-sectional study in Suzhou, China. Options VIII for the control of influenza Cape Town, South Africa: ISIRV, 2013:99.

16. Bu L, Hao X, Bo T, et al. Surveying the medical professionals' knowledge, attitude and practice of influenza and influenza vaccine in Qingdao. The Chinese Health Service Management 2015;32:474-6

17. Ortiz JR, Englund JA, Neuzil KM. Influenza vaccine for pregnant women in resource-constrained countries: a review of the evidence to inform policy decisions. Vaccine 2011;29:4439-52.

18. Suzhou Statistic Bureau. Suzhou statistical yearbook: China Statistics Press, 2016.

19. Dai L. Effects of prenatal care and gestational weight gain on pregnancy outcomes using restricted cubic spline [Master]: Suzhou University, 2015

20. Suzhou Health Bureau. Public health statistic report in Suzhou in 2016 2017. http://www.zfxxgk.suzhou.gov.cn/sjjg/szswshjhsywyh/ 201705/t20170511_868619.html

21. Wang J. In: Zhang J, ed. Suzhou maternal and child health care service, 2015.

22. Centers for Disease Control and Prevention. Influenza specimen collection. 2017 http://www.cdc.gov/flu/pdf/freeresources/ healthcare/flu-specimen-collection-guide.pdf

23. National Health and Family Planning Committee. National influenza surveillance protocol. 2010 http://www.nhfpc.gov.cn/jkj/s3577 /201009/3fa356d0f4834d408fde6c12891a6482.shtml (cited 8 May 2017).

24. He X. The R \& D and application of maternal and child health information management system of Suzhou city. Chin J Women Child Health 2012;3:109-11.

25. World Health Orgnization. WHO surveillance case definitions for ILI and SARI 2014. http://www.who.int/influenza/surveillance monitoring/ili_sari_surveillance_case_definition/en/ (cited 8 May 2017)

26. Chen L, Zhou S, Zhang Z, et al. Active surveillance for respiratory illness associated with influenza among pregnant women in Suzhou, China, 2015-2016. Options IX for the control of influenza Chicago, Illinois, USA: ISIRV, 2016:177-8. 